\title{
Chain Reorientation in $\beta$-PVDF Films Upon Transverse Mechanical Deformation Studied by SEM and Dielectric Relaxation
}

\author{
R. BARBOSA, ${ }^{1}$ J. A. MENDES,${ }^{1}$ V. SENCADAS,${ }^{1}$ J. F. MANO,${ }^{2}$ \\ and S. LANCEROS-MÉNDEZ ${ }^{1, *}$ \\ ${ }^{1}$ Dept. de Física, Universidade do Minho, 4710-057 Braga, Portugal \\ ${ }^{2}$ Dept. de Eng. de Polímeros, Universidade do Minho, 4800-058 Guimarães, Portugal
}

(Received September 4, 2002; In final form December 15, 2002)

\begin{abstract}
Chain reorientation may be induced in polyvinylidene fluoride (PVDF) in its $\beta$-phase by applying a deformation perpendicular to the pre-oriented polymeric chains. This reorientation begins right after the yielding point and seems to be completed when the stress-strain curve stabilizes. We have studied the chain reorientation mechanism by scanning electron microscopy (SEM) and dielectric relaxation methods and we have analyzed its influence in the $\alpha$ - and $\beta$-relaxations of the polymer. SEM images confirm the existence of a reorientation upon transversal deformation. The decrease of crystallinity with increasing reorientation and the dependence of the dielectric permittivity upon deformation indicate that a model of stress-induced melting followed by an incomplete recrystallization properly describes the reorientation mechanism.
\end{abstract}

Keywords: PVDF; dielectric relaxation; mechanical deformation; SEM

\section{INTRODUCTION}

Poly(vinilidene fluoride), PVDF, is known for its piezo-, pyro-, and ferroelectric properties. These properties are exploited in a number of applications such as sensors of heat, pressure, sound, etc. In many of these applications the material may be subjected to severe temperature changes or mechanical stresses. A strong mechanical stress dependency of the piezoelectric coefficient has been detected [1,2]. Under certain circumstances, the piezoelectric response can even change its sign.

One crucial problem is the dependence of these particular electrical properties on the structure of PVDF. One can assume that the distribution of

*Corresponding author. E-mail: lanceros@ fisica.uminho.pt 
lamella thickness, the degree of perfection of the two-phase structure, and the orientation of the chain elements in the amorphous region of PVDF are structural parameters, which also influence the electrical response and its temperature dependence. Such investigations are complicated since PVDF alters its crystal modification under thermal or stress treatment $[3,4]$. Of the five known modifications of PVDF, the $\alpha$ - and $\beta$-modifications are the most common. The $\alpha$-modification arises usually at crystallization from the melt. It is transformed into the $\beta$-modification by stretching the material by a ratio of about four and poling. Consequently, unpoled $\alpha$-crystalline material is usually isotropic, whereas PVDF in the $\beta$-modification is highly oriented [4].

Further, it has been concluded that the interphase between the crystalline and amorphous phases is of eminent importance for the polarization in PVDF [2]. In this interphase, a stress dependent orientation correlation of the molecular electric moments can exist, which in turn is induced by the complete orientation correlation of the moments in the crystalline phase, particularly in $\beta$-crystalline material. It randomizes only in the amorphous bulk. Estimations made by means of computer simulations of the chain conformation in this interphase show that its thickness amounts to about $1 \mathrm{~nm}$. At usual supermolecular structure, particularly, at crystallinity around 50\%, the amorphous phase carries, by means of this interphase, an average polarization of $20 \mathrm{mC} / \mathrm{m}^{2}$, i.e. about one third of the overall polarization. Considering the extensibility of the amorphous phase with stress and with change of temperature, it is obvious that the structure and the dynamic of the amorphous phase will play an important role in the ferroelectricity, piezoelectricity and pyroelectricity of this material [2].

In this investigation, we have extended previous DSC and FTIR studies on the dependence of $\beta$-PVDF upon a transversal deformation [5, 6]. We have investigated the dependence of the dielectric permittivity on the material structure at different mechanical stresses and temperatures, with especial attention to the behavior of the $\alpha$ - and $\beta$-relaxations and the high temperature shrinkage and conductivity. SEM was also used in order to get information on the surface texture of the material and its changes upon transversal deformation.

\section{EXPERIMENTAL}

The starting material was a $28 \mu \mathrm{m}$ thick commercial $\beta$-PVDF film from Measurements Specialties, Inc. (Fairfield, NJ, USA). Some samples were prepared by controlled deformation at different degrees (from $18 \%$ to break (max. 140\%)) in the perpendicular direction to the initial chain orientation 
[4]. For this preparation, Instron 4505 universal testing machine in the tensile mode at test velocities of $\mathrm{dl} / \mathrm{dt}=2 \mathrm{~mm} / \mathrm{min}$ in $8 \mathrm{~cm} \times 1 \mathrm{~cm}$ rectangular samples was used.

Scanning Electron Microscopy (SEM) was carried out at a resolution of $20 \mu \mathrm{m}$ in a Leica Cambridge apparatus. Samples with permanent deformations of $0 \%, 30 \%$ and $67 \%$ were investigated.

Dielectric measurements were performed in the same samples with a General Radio 1693 digital RLC bridge. The real $\left(\varepsilon^{\prime}\right)$ and imaginary $\left(\varepsilon^{\prime \prime}\right)$ parts of the permittivity were obtained in the frequency range $100 \mathrm{~Hz}$ to $100 \mathrm{kHz}$, at a temperature rate of $1 \mathrm{~K} / \mathrm{min}$, from 77 to $414 \mathrm{~K}$.

\section{EXPERIMENTAL RESULTS}

\subsection{Scanning Electron Microscopy}

Three samples in the three different regions of the stress-strain diagram were analyzed (Fig. 1): a virgin sample with no mechanical deformation;

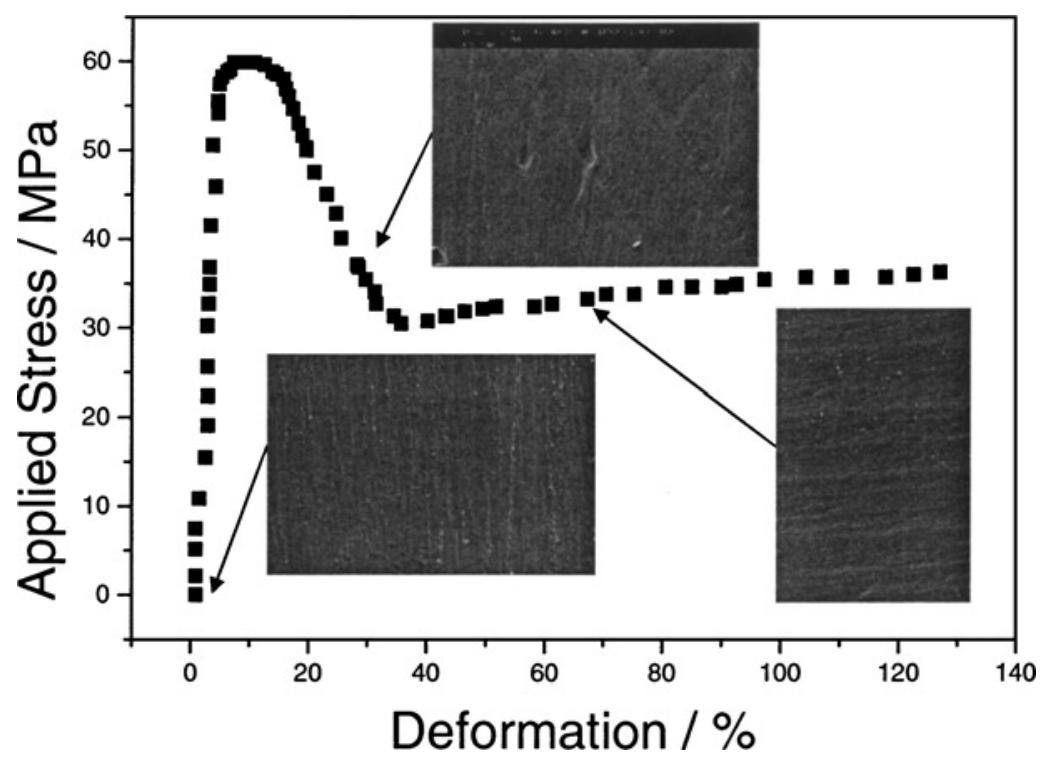

Figure 1. SEM results for $\beta$-PVDF samples with permanent deformations $(0 \%$, $30 \%$ and $67 \%$ ) in the three main regions of the stress-strain curve. The orientation of the images corresponds to the same orientation of the samples: perpendicular to direction of the applied stress. The stress was applied perpendicularly to the original draw direction. Resolution of $20 \mu$. 
a sample with a permanent deformation of $\sim 30 \%$, i.e. in the intermediate region between the yielding point and the plateau region; and a sample with a permanent deformation of $\sim 67 \%$, i.e. in the plateau region of the stress-strain diagram.

The anisotropic nature of the $\beta$-PVDF films is confirmed by the SEM images by showing a fibrilar structure that is observed in the three samples. On the other hand, whereas in the virgin sample the fibrilar structure is parallel to the draw direction, in the sample with a deformation of $\sim 67 \%$ this structure has rotated to a direction perpendicular to the original one and parallel to the direction in which the mechanical stress is being applied. The sample with a deformation of $\sim 37 \%$ shows an intermediate situation, with the fibrilar structures slightly tilted with respect to the original orientation. These results are in agreement with previous FTIR results [5, 6].

\subsection{Dielectric Spectroscopy}

Dielectric relaxation experiments were performed in previously deformed samples in order to evaluate the effect of the chain reorientation both in the $\varepsilon^{\prime}$ and in the different relaxations observed in this material.

The results obtained for the virgin sample [6] reveal the low temperature $\beta$-relaxation, assigned to the glass transition dynamics, but no $\alpha$-relaxation is observed at higher temperatures, associated with molecular motions within the crystalline fraction. An increase of $\tan \delta$ and a shrinking of the sample are observed instead $[6,7]$.

When a stress is applied to the sample, the structural changes are reflected in the behavior of the dielectric permittivity, both with temperature and frequency. Figure 2 shows the temperature and frequency behavior of the dielectric permittivity for the sample with a deformation of $30 \%$. A comparison between $\varepsilon^{\prime}(\mathrm{T})$ and $\tan \delta(\mathrm{T})$ at a frequency of $10 \mathrm{kHz}$ for the samples in the three regions of the stress-strain curve is shown in Fig. 3. The values of various relevant dielectric parameters for the three samples are summarized in Tables I and II.

The main features of the dielectric behavior of the unstressed sample are preserved in the samples with permanent deformation, though important changes occur in the overall values of $\varepsilon^{\prime}$ and $\tan \delta$, in particular, in the regions of the $\alpha$ - and the $\beta$-relaxations.

The value of $\varepsilon^{\prime}$ at $275 \mathrm{~K}$ (i.e. above the $\beta$-relaxation) suffers a linear decrease for samples with increasing deformation (Table I): from 8.39 for the virgin sample to 4.29 at a deformation of $67 \%$. The analysis of the decrease in $\varepsilon^{\prime}$ by lowering temperature was analysed by considering the 


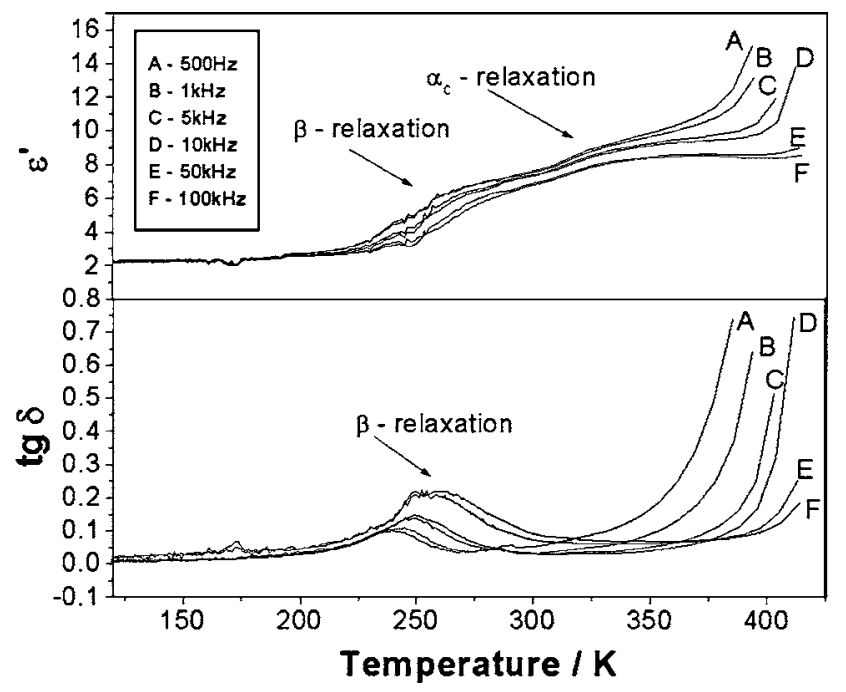

Figure 2. $\varepsilon^{\prime}$ (above) and $\tan \delta$ (below) versus temperature for $\beta$-PVDF with a permanent deformation of $30 \%$, measured at several frequencies between $500 \mathrm{~Hz}$ (A) and $100 \mathrm{kHz}(\mathrm{F})$.

value of $\varepsilon^{\prime}$ before $(\mathrm{T}=275 \mathrm{~K})$ and after $(\mathrm{T}=200 \mathrm{~K})$ the $\beta$-relaxation, and normalizing by the corresponding value at $275 \mathrm{~K}$. By comparing these values for the three samples we concluded that the decrease of $\varepsilon^{\prime}$ accompanying the $\beta$-relaxation is almost constant till the sample enters the plateau region of the stress-strain curve, when this value is heavily reduced (Table I). A
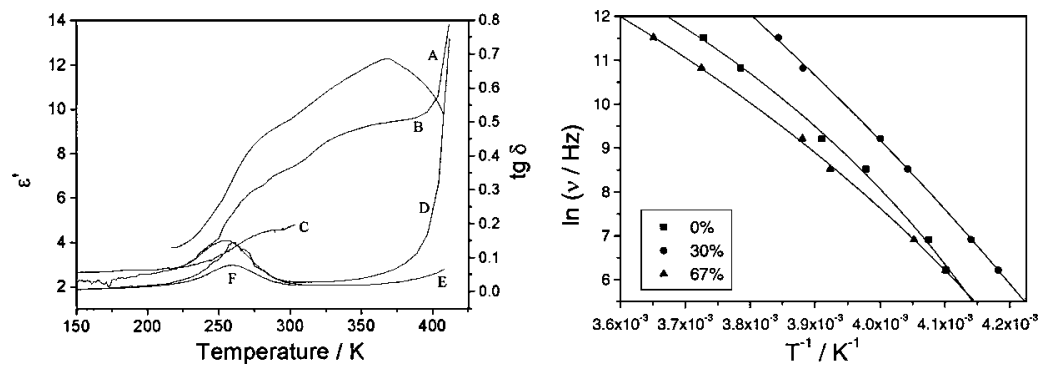

Figure 3. Left: comparison of the $\varepsilon^{\prime}(\mathrm{T})$ results for samples with three permanent deformations: A-0\%; B-30\%; C-67\% and comparison of the $\tan \delta(\mathrm{T})$ results for the same samples: D-0\%; E-30\%; F-67\%, at a fixed frequency of $10 \mathrm{kHz}$. Right: Vogel-Tammann-Fulcher fits of the $\beta$-relaxation of $\beta$-PVDF for the three samples. 
TABLE I Relevant dielectric parameters for $\beta$-PVDF samples with three permanent transversal deformations (\%). The values are given for a frequency of $10 \mathrm{kHz}$

\begin{tabular}{rccccc}
\hline$\%$ & $\varepsilon^{\prime}(275 \mathrm{~K})$ & $\Delta \varepsilon^{\prime} / \varepsilon^{\prime}$ & $\operatorname{tg} \delta_{\max }$ & $\mathrm{T}\left(\operatorname{tg} \delta_{\max }\right) / \mathrm{K}$ & $\varepsilon^{\prime \prime}$ \\
\hline 0 & 8.48 & 0.64 & 0.151 & 254.85 & 0.70 \\
30 & 6.35 & 0.70 & 0.148 & 261.43 & 0.48 \\
67 & 4.30 & 0.39 & 0.079 & 259.43 & 0.23 \\
\hline
\end{tabular}

similar behaviour is observed in the analysis of $\tan \delta$ in the region of the $\beta$-relaxation: whereas the value of the maximum $\tan \delta(\mathrm{T})$ is almost constant for the samples with $0 \%$ and $30 \%$ deformation, the reduction of the dielectric loss for deformations larger than $40 \%$ is very pronounced (Table I). The temperature of this maximum, on the other hand, suffers an increase for the deformed samples, being this increase slightly larger for the $30 \%$ sample (Table I)

The dynamic of the $\beta$-relaxation was analyzed by the Vogel-TammannFulcher relaxation formalism

$$
\tau=\tau_{0} \exp \left(\mathrm{E}_{\mathrm{VTF}} / \mathrm{k}\left(\mathrm{T}-\mathrm{T}_{\mathrm{o}}\right)\right)
$$

where $\mathrm{E}_{\mathrm{VTF}}$ is the VTF energy, $\mathrm{k}$ is the Boltzmann constant and $T_{0}$ is the critical temperature at which molecular motions in the material become infinitely slow. The parameters obtained for the different samples are summarized in Table II.

An important increase of the activation energy for the sample in the postyielding region, accompanied by a decrease of the Vogel-Tamman-Fulcher temperature was observed. By further increasing of the stress and reorientation of the samples, these values return to intermediate values between those of the virgin and the $30 \%$ deformed sample (Table II).

TABLE II Vogel-Tammann-Fulcher and fragility parameters for the $\beta$-relaxation of $\beta$-PVDF samples with three permanent transversal deformations $(\%)$

\begin{tabular}{rcccc}
\hline$\%$ & $\tau_{0} / \mathrm{s}$ & $\mathrm{E}_{\mathrm{VTF}} / \mathrm{eV}$ & $\mathrm{T}_{\mathrm{VTF}} / \mathrm{K}$ & $\mathrm{m}$ \\
\hline 0 & $5.05 \mathrm{E}-10$ & 0.061 & 196.54 & 92.26 \\
30 & $3.33 \mathrm{E}-16$ & 0.247 & 141.16 & 52.14 \\
67 & $3.26 \mathrm{E}-11$ & 0.110 & 172.72 & 61.05 \\
\hline
\end{tabular}


A consequence of the values of the fitting parameters of the VTF relaxation plot is the calculation of the fragility parameter [6]:

$$
m=\frac{\mathrm{E}_{\mathrm{VTF}} / k T_{g}}{(\ln 10)\left(1-T_{0} / T_{g}\right)^{2}},
$$

where $m$ is an indication of the steepness of the variation of the material properties (viscosity, relaxation time...) as $T_{g}$ is reached. A high $m$ value defines a fragile material whereas a strong one will be characterized by small $m$ values.

The values of several glass-forming amorphous polymers range from 46 (polysobutylene) to 191 (polyvinylchloride) [8]. The $m\left(\mathrm{~T}_{\mathrm{g}}\right)$ value calculated with the VTF parameters for $\beta$-PVDF is 92 determined at the glass transition temperature $\left(\mathrm{T}_{\mathrm{g}}\right)$ where the relaxation time is equal to $100 \mathrm{~s}$. The fragility parameters of $\alpha$ - and $\beta$-PVDF are very similar. This demonstrates that only the amorphous part of the material is involved in this relaxation process. On the other hand, the increase of deformation produces an important decrease of the fragility parameter $m\left(\mathrm{~T}_{\mathrm{g}}\right)=52$ for the $30 \%$ deformed sample, i.e. the material becomes stronger. Again, when the material enters the plateau region, the parameter return to intermediate values between the virgin and the $30 \%$ deformed sample $m\left(\mathrm{~T}_{g}\right)=61$ for the $67 \%$ deformed sample.

The main difference between the stretched and the virgin samples occurs in the region of the $\alpha$-relaxation. In the virgin sample, an increase in tan $\delta(\mathrm{T})$ starting at $\mathrm{T} \sim 336 \mathrm{~K}$ and an anomalous decrease of $\varepsilon^{\prime}(\mathrm{T})$ at $\sim 364$ $\mathrm{K}$ is observed (Fig. 3a). There is no hint of the $\alpha$-relaxation, which is observed by mechanical relaxation [7]. On the other hand, the samples with permanent deformation do not show the decrease in $\varepsilon^{\prime}(\mathrm{T})$. Instead, an almost exponential increase of $\varepsilon^{\prime}(\mathrm{T})$ accompanied by frequency dispersion is observed, i.e. perpendicular deformation prevents the shrinkage of the samples. In these samples, a hump in the dielectric constant around $334 \mathrm{~K}$ indicates the $\alpha$-relaxation.

At $\mathrm{T} \geq 345 \mathrm{~K}$ the frequency dependence of the real (imaginary) part of the complex permittivity of $\beta$-PVDF can be fitted by equation 3 :

$$
\varepsilon^{*}=(v)=\varepsilon_{\infty}+B \operatorname{Im}(i 2 \pi v)^{\mathrm{a}}
$$

where $\varepsilon_{\infty}$ is the high frequency limit of the real part of the dielectric permittivity which corresponds to the static permittivity of the nonconductive crystal, $B$ and $a$ are constants (Fig. 4). The obtained values (e.g. $\varepsilon_{\infty}^{\prime}=4.36$; $B=2.48 ; a=0.14$ for $\mathrm{T}=348 \mathrm{~K}$ ) are typical for hopping conduction in 


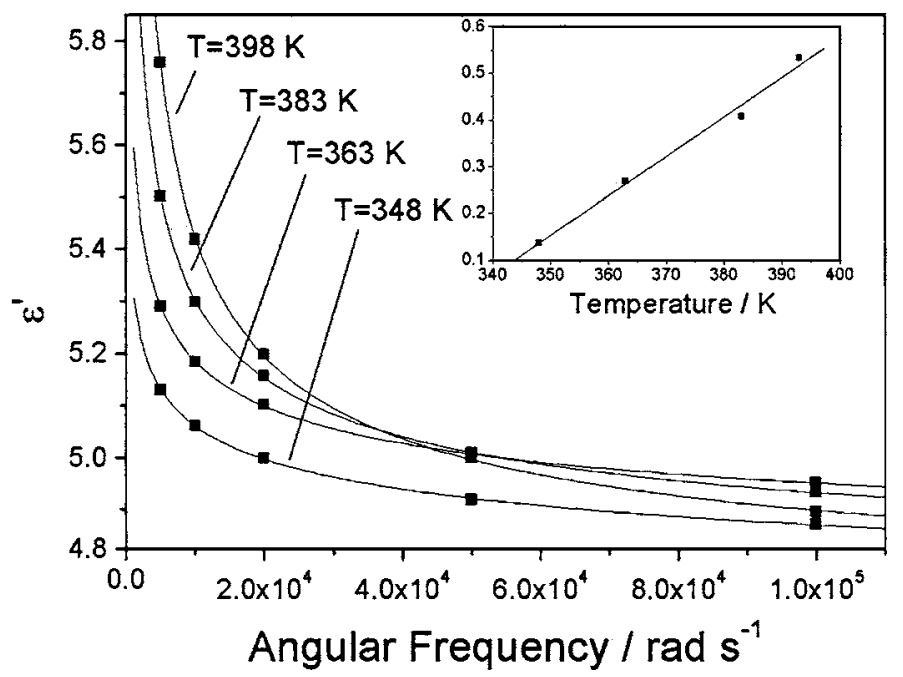

Figure 4. Real part of the complex dielectric function as a function of frequency for $\beta$-PVDF with a permanent transversal deformation of 30\%. Lines are fits to Eq. 3 . Inset: approximate power-law exponent as a function of temperature.

disordered media [9]. These free charges could be ionic species from chemical additives used for polymerization as well as dissociated water into the polymer. The fitting results are similar to the ones obtained in the virgin sample, though there is a slight increase of the conductivity for the samples in the post-yielding region (Fig. 3). The configurational changes accompanying the deformation process do not seem to influence the conduction mechanisms, but the number of charge carriers and/or their mobility. The differences obtained between the undeformed and the deformed samples in the higher temperature region are due to the existence of shrinkage in the undeformed samples [7].

\section{DISCUSSION}

There are a number of approaches to the effect of stretching chains on the relaxation mechanism [10]. The configuration entropy ideas leading to the prediction of a second order transition can be used to give a clear prediction of $T_{g}$ trend with extension, provided $T_{g}$ trends parallel to the second order transition temperature. If a chain is extended from its unperturbed random coil state the configurational entropy decreases. Thus on cooling this 
entropy becomes zero at a higher temperature and $T_{g}$ is predicted to increase. Conversely the predictions from Eyring's equation based on stress activation predict the opposite trend [10]. This later behavior is the observed one in our samples, in relation to the $\beta$-relaxation.

In semicrystalline polymers, yielding involves considerable disruption of the crystal structure. Slip occurs between the crystalline lamellae, which slide by each other; and within the individual lamellae. The latter process is the dominant one, and leads to molecular orientation, since the slip direction within the crystal is along the axis of the molecule. As plastic flow continues, the slip direction rotates systematically towards the tensile axis. Ultimately, the slip direction (i.e. the molecular axis) coincides with the tensile axis, and the polymer is then oriented and resists to further extension.

Previous FTIR experiments showed a polarisation switching when the material was subjected to tensile stress perpendicular to the draw direction $[5,6]$. A detailed investigation of the post-yielding region showed that this switching occurs progressively within this region [6]. DSC in the stretched samples did not exhibit different crystalline phases, but a decrease in the degree of crystallinity upon deformation with no changes in the crystalline lamellae thickness [6].

These previous results, together to the new SEM and dielectric results, point out to a model of plastic deformation occurring by fusion and incomplete recrystallisation of the crystal phase present in the semicrystalline polymer [11]. This can account for the dynamic behaviour of the dielectric parameters, in which stronger variations of the values of, e.g. $\mathrm{E}_{\mathrm{VTF}}, T_{0}$ and $m$ with respect to the unstrained sample are obtained in the transitional postyielding region, to return to intermediate values when the material enters the plateau region, where a similar microstructure with respect to the virgin sample is recovered, but with a lower degree of crystallinity and a lower dielectric response, as indicated by the behaviours of $\varepsilon^{\prime}$ and $\tan \delta$.

The variations observed in the dielectric permittivity pointed out a participation of the amorphous region in the deformation process. Though the chains in the amorphous bulk state could be hardly associated to the process leading to permanent deformation, as their relaxation times are very small at room temperature, the presence of trapped entanglements among such segments could contribute as load transfer for permanent deformation. The interface region, i.e. the amorphous region related to the tie-chains that directly links adjacent crystalline lamellae and tie-chains linking the crystalline and the amorphous phases are expected to be highly taut and highly constrained and will dominate the mechanical behaviour in the plastic region. The activation of such tie-chain may contribute to change the orientation 
of the crystalline lamellae and have a strong influence in the absence of shrinkage and in the dielectric behaviour of the deformed samples.

\section{CONCLUSION}

A chain reorientation is confirmed in $\beta$-PVDF upon transversal deformation that affects the dielectric properties of the material. The $\beta$-relaxation is identified in the virgin as well as in the deformed samples. On the other hand, the $\alpha$-relaxation is only detected in the deformed samples, due to the absence shrinkage upon heating. The high temperature conductivity can thus be ascribed to hopping conductivity due to free charges induced during poling. The configurational changes that occur upon transversal deformation increase the high temperature conductivity but seem to have no influence in the conduction mechanisms.

In the case of the studied oriented $\beta$-PVDF film, we expect that the tiechains of the interphase will dominate the plastic behavior of the material and deeply influence the dielectric response of the deformed samples.

The decrease of crystallinity and the behavior of the dielectric parameters upon transversal deformation support the model of melting and incomplete recrystallization as the mechanisms underlying the reorientation process $[6,11]$.

\section{ACKNOWLEDGEMENT}

Work supported by the Portuguese Foundation for Science and Technology under Grant POCTI/CTM/33501/99.

\section{REFERENCES}

[1] B. R. Hahn, J. Appl. Phys. 57, 1294 (1985).

[2] J. H. Vinson and B. J. Jungnickel, Ferroelectrics 216, 63 (1998).

[3] T. Furukawa, Phase Transitions 18, 143 (1989).

[4] A. J. Lovinger, Developments in Crystalline Polymers, Vol. 1, ed. D. C. Basset (London, Elsevier Applied Science, 1982).

[5] S. Lanceros-Mendez, J. F. Mano, A. M. Costa, and V. H. Schmidt, J. Macrom. Sci.- Phys. B40, 517 (2001).

[6] S. Laceros-Mendez, M. V. Moreira, J. F. Mano, V. H. Schmidt, and G. Bohannan, Ferroelectrics 273, 15 (2002).

[7] V. Sencadas, R. Barbosa, J. F. Mano, and S. Lanceros-Méndez, Ferroelectrics, this issue. 
[8] R. Bohmer, K. L. Ngai, C. A. Angell, and D. J. Plazek, J. Chem. Phys. 99, 4201 (1993).

[9] J. C. Dyre and T. B. Schroder, Rev. Mod. Phys. 72, 3 (2000).

[10] R. E. Wetton, Eur. Polym. J. 29, 131 (1993).

[11] S. Hellinckx and J. C. Bauwens, Colloid Polym. Sci. 273, 219 (1995). 